\title{
Finding critical points using improved scaling Ansätze
}

\author{
M Roncaglia ${ }^{1}$, L Campos Venuti ${ }^{2}$, C Degli Esposti Boschi ${ }^{3}$ \\ ${ }^{1}$ INRIM, Strada delle Cacce 91, 10135, Torino, Italy \\ Politecnico di Torino, Corso Duca degli Abruzzi 24, 10129 Torino, Italy \\ ${ }^{2}$ University of Southern California, Los Angeles, CA 90089, USA \\ 3 CNR-IMM, Sezione di Bologna, via Gobetti 101, 40129, Bologna, Italy
}

\begin{abstract}
Analyzing in detail the first corrections to the scaling hypothesis, we develop accelerated methods for the determination of critical points from finite size data. The output of these procedures is sequences of pseudo-critical points which rapidly converge towards the true critical points. The convergence is faster than that obtained with the fastest method available to date, which consists of estimating the location of the gap's closure (the so called phenomenological renormalization group). Having fast converging sequences at our disposal, allows us to draw conclusions on the basis of shorter system sizes. This can be extremely important in particularly hard cases such as two-dimensional quantum systems with frustrations or in Monte Carlo simulations when the sign problem occurs. After reviewing the most efficient techniques available to date, we test the effectiveness of the proposed methods both analytically on the basis of the one-dimensional XY model, and numerically at phase transitions occurring in non integrable spin models. In particular, we show how a new Homogeneity Condition Method is able to produce fast converging sequences in correspondence of the Berezinskii-KosterlitzThouless (BKT) transition simply making use of ground-state quantities on relatively small systems. Remarkably, our method tested on the frustrated spin-1/2 Heisenberg model gives a BKT critical point which is incompatible with the ones present in the past literature, based on different methods. This discrepancy raises the fundamental question to determine the correct renormalization group approach and scaling assumptions that yield to the sequences converging to the true critical point. Finally, we formulate a general prescription that allows to analyze and efficiently locate critical points in a variety of cases, without knowing in advance the universality class of the tested transition. Even if our methods are here tested in one dimension, we expect them to be valid in any spatial dimensionality and both for quantum and classical statistical systems.
\end{abstract}

PACS numbers: 05.70.Fh, 64.60.an, 75.40.Mg 


\section{Introduction}

In statistical mechanics the exact evaluation of equilibrium properties, except for non-interacting systems, can be worked out only in very few notable integrable models [6]. Thanks to the increasing power of computer resources, numerical simulations on finite size systems are becoming a more and more popular. Among them we quote exact diagonalization (ED) algorithms, density matrix renormalization group (DMRG), and (quantum) Monte Carlo methods. The first question one asks in this context is the determination of critical points in order to classify the phase diagram into different phases. Once critical points are found, one can ask what is the nature of the critical point by computing critical exponents. Though statistical mechanics was born more than a hundred years ago, and its theoretical understanding is now on a sound basis after the scaling hypothesis and the theory of renormalization group, until now only somehow phenomenological methods are available for the determination of critical points from finite size data. In this article we carefully analyze the leading corrections of equilibrium averages beyond the usual scaling hypothesis. In this way we are able to find accelerated methods for the determination of critical points. The major advantage of having faster converging methods is that one can content oneself with simulations on shorter sizes. Such simulations are those which can be obtained with higher accuracy in favorable cases, but are the only one available in the most demanding situations given for instance by frustrated quantum systems in two dimensions.

Traditionally classical (some would say Euclidean) statistical systems, have been studied by signaling out an order parameter (when present). An example can be the magnetization in a magnetic system $\langle M\rangle$, which, in the thermodynamic limit, is non zero only in the ordered phase, and so allows to locate precisely the critical point. This is only true in the thermodynamic limit, instead at finite sizes the situation is less clear, and typically the magnetization will be different from zero everywhere, presenting "finite size tails" in the paramagnetic region. A possible, widely used, strategy is that of concentrating on susceptibilities, i.e. derivative of the magnetization. It can happen that the susceptibility diverges at the critical point in the infinite volume limit. At finite size singularity are smoothed and the susceptibility only displays a maximum at a certain point which is shifted respect to the actual critical point. Of course when one considers larger and larger sizes, the pseudo-critical point given by the maximum of the susceptibility, shifts towards the true critical point. The convergence rate is algebraic in the system size $L$ and is of the form $L^{-\theta}$ defining the so called shift exponent $\theta$. This method of course is limited to those cases where a susceptibility diverges. A somewhat more elaborated method is that of the Binder's cumulant [7].

In this approach one considers the fourth order reduced cumulant $U_{L} \equiv 1-\left\langle(\Delta M)^{4}\right\rangle /\left[3\left\langle(\Delta M)^{2}\right\rangle^{2}\right]$ where $\Delta M=M-\langle M\rangle$, in a finite system of linear size $L$. Since by the scaling hypothesis $U_{L}$ should depend only on the ratio $L / \xi$ where $\xi$ is the correlation length, at the critical point $\xi \rightarrow \infty$ and $U_{L}$ should be independent of $L$. The finite size pseudo critical point is then obtained by solving $\partial_{L} U_{L}=0$ (here and in the following, at finite size, derivatives with respect to $L$ must be interpreted as finite differences). Graphically this amounts to finding the crossing points of the curves $U_{L}$ at successive values of $L$.

In quantum statistical systems the method of choice is given by the so called phenomenological renormalization group (PRG) [4, 15]. Continuous phase transitions in quantum mechanics are characterized by closure of the first gap $\Delta_{L}$. Scaling hypothesis then asserts that the gap must be a function of $\xi$ hence $\Delta_{L} \sim \xi^{-\zeta}$ which defines the dynamical exponent $\zeta$. Since at the critical point the correlation length will be of the order of the system size, the quantity $L^{\zeta} \Delta_{L}$ will be independent 
of $L$. The PRG equation is then given by $\partial_{L}\left[L^{\zeta} \Delta_{L}\right]=0$.

All these methods produce a sequence of pseudo critical points, say $T_{L}^{*}$ which scale to the critical point according to an algebraic law $T_{L}^{*}=T_{c}+O\left(L^{-\theta}\right)$ governed by the shift exponent $\theta$. The general belief is that the shift exponent $\theta$ is given by the inverse of the correlation length exponent $\nu$, as it happens to the maximum of susceptibility. Although, to our knowledge, no systematic study of shift exponents has been done, it was already pointed out in [11] that the relation $\theta=1 / \nu$ is not always valid, and $\theta$ depends, among other factors, on the boundary conditions. In particular it should be clear that $\theta$ depends on the specific way in which the pseudo-critical point has been obtained.

It should be reminded here that in recent times new tools for the characterization of quantum critical points, inherited from the theory of quantum information, have arisen [3]. Among these the so called fidelity approach to phase transition consists of looking for the maximum of the so called quantum Fisher information [9, 22]. This method is the best possible one if the only available resource we admit is measuring observables. As we will see we can do much more, i.e. compare different observables, compute finite difference and so on. In any case it was shown in [23] that, except for accidental cancellations, the shift exponent obtained with this method is $\theta=1 / \nu$.

The lesson we should bear from this short resumé, is twofold. On the one hand the present methods for the location of critical point are somehow phenomenologically obtained assuming/imposing the scaling hypothesis. On the other hand scaling hypothesis alone would imply that finite size pseudocritical points actually coincide with critical points. To learn about the shift exponents and to develop faster converging sequences one must go beyond the usual scaling hypothesis.

\section{Finite size scaling beyond scaling hypothesis}

To be concrete we will deal with quantum systems in $d$ spatial dimension at zero temperature endowed with periodic boundary conditions (PBC) to get rid of border effects. Our results can however be easily generalized to finite temperature (in which case the role of the energy will be played by the free energy) and/or classical statistical mechanics in $D$ dimension (and use the dimensional crossover rule $D \leftrightarrow d+\zeta$ ). The Hamiltonian can be written as

$$
\mathcal{H}=\mathcal{H}_{0}+g \mathcal{V}=\mathcal{H}_{1}+\left(g-g_{c}\right) \mathcal{V}
$$

At the critical point $g_{c}$ the model is given by the Hamiltonian $\mathcal{H}_{1}=\mathcal{H}_{0}+g_{c} \mathcal{V}$ which, by hypothesis, is critical and so defines a scale invariant theory. The perturbation $\mathcal{V}$ can then be expanded in terms of the scale-invariant operators of the theory $\mathcal{H}_{1}$, this is the so called hypothesis of local algebra [17]. These operators are classified into relevant/irrelevant in case their scaling dimensions are smaller/larger than $d+\zeta$, the case $d+\zeta$ being marginal. Since, for $g \neq g_{c}$ the model is not critical, there must be at least one relevant component in $\mathcal{V}$ which is responsible for driving the system away from criticality. This simple observation motivates the fact that, at least in principle, it should be possible to estimate the critical point, only considering the finite size sequence of the perturbation operator $b_{L} \equiv\langle\mathcal{V}\rangle / L^{d}$. Such a strategy has been advocated in [8], where the so called finite size crossing method (FSCM) has been developed. It was found that a sequence of pseudocritical points $g_{L}^{*}$ could be given by the simple FSCM equation $\partial_{L} b_{L}\left(g_{L}^{*}\right)=0$. As we will see later this method results in a shift exponent twice as large as the "standard" one: $\theta_{F S C M}=2 / \nu$. 
The energy density $e=\langle\mathcal{H}\rangle / L^{d}$ can be split into a regular and a singular part: $e=e_{\text {reg }}+e_{\text {sing. }}$. In the off-critical regime $L \gg \xi$ the singular part behaves as

$$
e_{\text {sing }} \sim|t|^{2-\alpha}
$$

where $t=g-g_{c}$. In the most general case, the coefficient in front of Eq. (11) can depend on the side the transition is approached, i.e. $e_{\text {sing }} \simeq A_{ \pm}|t|^{2-\alpha}$ according to whether $t \rightarrow 0^{ \pm}$. Scaling hypothesis asserts that, in a finite system of linear size $L$, thermodynamic averages depend only on the ratio $L / \xi$, where, close to the transition point, $\xi \sim|t|^{-\nu}$. Since for finite systems averages must be analytic in $g$, the singular part of the energy must effectively depend on the scaling variable $z=t L^{1 / \nu}$. So we arrive at

$$
e_{\text {sing }}=L^{-(d+\zeta)} \Phi_{0}(z)+\cdots,
$$

where the dots represent corrections arising from less singular contributions. Here $\Phi_{0}(z)$ is a universal scaling function that for $z \rightarrow \pm \infty$ (the off-critical regime) must behave as $\Phi_{0}(z) \sim$ $A_{ \pm} z^{(d+\zeta) \nu}$ in order to recover Eq. (1). Comparing with Eq. (10) one finds $2-\alpha=(d+\zeta) \nu$. Instead, for $L \ll \xi$ we are in the critical regime and $\Phi_{0}(z)$ behaves as an analytic function. The only requirement is that, at finite $L, \Phi_{0}$ must be such that $e$ is an analytic function of $g$ also around $g_{c}$. We will come back to this point later.

Equation (1) gives us the leading order singular part of the free energy. Obviously one can expect corrections to such leading behavior. One kind of corrections, so called analytic corrections, have the form:

$$
e_{\text {sing }} \sim|t|^{2-\alpha}\left(1+a_{1} t+a_{2} t^{2}+\cdots\right) .
$$

Analytic corrections can be simply accounted for by noting that the scaling variable $z$ is only approximately $z \approx t L^{1 / \nu}$. Using a more accurate prescription we can write $z=$ $t\left(1+b_{1} t+b_{2} t^{2}+\ldots\right) L^{1 / \nu}$. Setting this "improved" scaling variable $z$ into Eq. (2) we obtain Eq. (3) with $a_{1}=b_{1}(2-\alpha)$ and $a_{2}=(2-\alpha)\left(2 b_{2}+b_{1}^{2}(1-\alpha)\right) / 2$ [2]. This sort of analytic non-linearities are a consequence of the fact that the RG equations are only approximately linear at the fixed point.

Besides analytic corrections, we can have corrections to Eq. (11) arising from irrelevant terms. This terms contribute to the singular part of the free energy with an additive term of the form [21]

$$
B_{ \pm} h(t)|t|^{2-\alpha+\lambda}
$$

with $\lambda$ not an integer and $h$ an analytic function of $t$ in zero. Such terms can be accounted for by a scaling function of the form

$$
L^{-(d+\zeta)-\omega} h(t) \Phi_{1}(z) .
$$

In the off-critical regime $z \rightarrow \pm \infty$, we must have $\Phi_{1}(z) \sim B_{ \pm} z^{(d+\zeta+\omega) \nu}$ and we find $\lambda=\omega \nu$. Summarizing, the scaling form for the singular part of the free energy, including only the least irrelevant operator, is

$$
e_{\text {sing }}=L^{-(d+\zeta)}\left[\Phi_{0}(z)+L^{-\omega} h(t) \Phi_{1}(z)\right] .
$$


In the computation of the shift exponents, other terms in the expansion of the energy density may play an important role. We write these terms as

$$
F(g) L^{-(d+\zeta)}+\ldots
$$

Where the function $F$ is analytic in $g$ also around $g_{c}$. Note that the next term in such an expansion would be of the form $M(g) L^{-(d+\zeta+\epsilon)}$, with $M(g)$ also analytic. Such a term, close to the critical point is equivalent to that arising from an irrelevant operator with $\omega=\epsilon$. Therefore we do not include such terms as they are already included in equation (4).

In $d=1$, when the theory is conformally invariant (and so $\zeta=1$ ), the origin of a $g$-dependent term of order $L^{-(d+\zeta)}$ like the one in (5), can be justified in the following way. In this case it is known that [1], at the critical point, the energy density behaves as $e=e_{\infty}\left(g_{c}\right)-L^{-2} \pi c v / 6+\cdots$. Here $c$ is the central charge and $v$ the speed of elementary excitations. Now, the effect of the perturbation, beside that of opening a gap, will be that of renormalizing the speed of elementary excitations $v \rightarrow v(g)$ giving rise to the function $F(g)=-\pi c v(g) / 6$. The presence of such terms can also be checked in the $X Y$ model via an explicit computation (see section 3.1). The appearance of a $g$-dependent Casimir term, analogous to our $F(g) L^{-(d+\zeta)}$, has also been demonstrated for the Casimir effect when adding a small mass to the electromagnetic field [5].

Armed now with equation (4) and possible corrections of the form (5), we can now compute shift exponents related to different techniques. The recipe is simple. What we have to do is expand the equation determining the finite-size pseudo critical point, in the quasi-critical region $L \ll \xi$. It suffice to expand the equations up to linear terms in $\left(g-g_{c}\right)$. Higher order terms in $\left(g-g_{c}\right)$ would result in corrections which decay more rapidly with $L$ and so can be safely ignored since we are interested in the leading behavior.

An important issue in the computation of shift exponents is whether the scaling function $\Phi_{0}$ (and/or $\left.\Phi_{1}\right)$ contain a linear term or not. If a linear term is present in, say $\Phi_{0}$, it means that the singular part of the energy will not be an even function of $t$ close to the critical point. As a consequence, different curves $e_{L}(g)$ at different sizes $L$ will cross close to the critical point. In other words one could apply the FSCM equation to the energy itself: $\partial_{L} e_{L}\left(g_{L}^{*}\right)=0$. However this method gives rise to a poor convergence rate, with a shift exponent given by $1 / \nu$ as usual. The same shift exponent is obtained if one applies the FSCM to the perturbation operator, i.e. solving $\partial_{L} b_{L}\left(g_{L}^{*}\right)=0$ as in the original formulation [8].

Let us consider in detail the equation $\partial_{L} e_{L}\left(g_{L}^{*}\right)=0$. Up to leading order the solution is given by

$$
g_{L}^{*}=g_{c}+\frac{(d+\zeta) \nu\left(F\left(g_{c}\right)+\Phi_{0}(0)\right)}{(1-(d+\zeta) \nu) \Phi_{0}^{\prime}(0)} L^{-1 / \nu} .
$$

We note that the leading convergence rate is dictated by the combination $\left(F\left(g_{c}\right)+\Phi_{0}(0)\right)$ being non-zero. In the energy density these terms correspond to quantities which scale as $L^{-(d+\zeta)}$ in the quasi-critical region. We now present a method which gets rid of such contributions and hence allows for a faster convergence rate.

\subsection{The homogeneity condition method}

Since the convergence rate in the FSCM is dictated by $\left(F\left(g_{c}\right)+\Phi_{0}(0)\right)$ being non-zero, one can seek for a method such that the terms of order $L^{-(d+\zeta)}$ cancels out exactly from the expression of 
$e_{L}$. This is achieved by requiring that the function $\partial_{L} e_{L}$ be homogeneous of degree $(d+\zeta+1)$. We arrive in this way at the homogeneity condition method (HCM), which consists of finding the solution to the following equation

$$
(d+\zeta+1) \partial_{L} e_{L}(g)+L \partial_{L}^{2} e_{L}(g)=0 .
$$

Taking into account the full Ansatz Eqs. (4) and (5) we find that the convergence rate of the pseudo-critical sequence so defined, at leading order is given by

$$
g_{L}^{*, H C M}-g_{c}=\frac{\nu^{2} \omega(d+\zeta+\omega) \Phi_{1}(0) h(0)}{[(d+\zeta) \nu-1] \Phi_{0}^{\prime}(0)} L^{-1 / \nu-\omega} .
$$

We see that the shift exponent in this case is $\theta_{H C M}=\omega+1 / \nu$. It should be pointed out that, by considering higher derivatives of $e_{L}$ with respect to $L$, the procedure could be iterated and one can in principle find even faster converging sequences. However then one would require explicit knowledge of other exponents which are actually unknown. Note also that, despite the origin of the HCM is that of eliminating a term of order $L^{-(d+\zeta)}$ from the expression of $e_{L}$, the HCM works irrespective of the presence of such a term in $e_{L}$.

The HCM equation (6) has been written using formally continuous value of $L$. To obtain the HCM condition at finite size we first replace derivatives with centered finite differences which have fast convergence properties, so $D_{L} e_{L} \equiv\left(e_{L+\delta L}-e_{L-\delta L}\right) /(2 \delta L)$ and $D_{L}^{2} e_{L} \equiv$ $\left(e_{L+\delta L}-2 e_{L}+e_{L-\delta L}\right) /(\delta L)^{2}$. Then in principle any combination which reduces to (6) for $L \rightarrow \infty$ would produce a pseudo-critical sequence with shift exponent given by $\theta_{H C M}$. However, at finite sizes, we can require that the term of order $O\left(L^{-(d+\zeta)}\right)$ in (5) cancels out exactly. This choice will result in sequences with nicer converging properties. This is achieved by choosing a proper combination of $D_{L} e_{L}$ and $D_{L}^{2} e_{L}$, and the HCM equation at finite size becomes

$$
P_{1}(L) D_{L} e_{L}(g)+P_{2}(L) D_{L}^{2} e_{L}(g)=0
$$

where the two polynomials $P_{1 / 2}$ are given by

$$
\begin{aligned}
& P_{1}(L)=\left[2 L^{d+\zeta}(L+\delta L)^{d+\zeta}+2 L^{d+\zeta}(L-\delta L)^{d+\zeta}-4\left(L^{2}-\delta L^{2}\right)^{d+\zeta}\right] \\
& P_{2}(L)=\delta L\left[L^{d+\zeta}(L+\delta L)^{d+\zeta}-L^{d+\zeta}(L-\delta L)^{d+\zeta}\right] .
\end{aligned}
$$

For instance, when $d+\zeta=2$ the HCM equation reduces to $\left[3 L^{2}-(\delta L)^{2}\right] D_{L} e_{L}(g)+L^{3} D_{L}^{2} e_{L}(g)=$ 0 , which correctly reduces to (6) in the limit of large $L$.

2.1.1. No linear terms in the scaling functions. As can be inferred by looking at equation (77), the $\mathrm{HCM}$ as is cannot be applied when the scaling function $\Phi_{0}$ does not contain a linear term. In this case one would have $\Phi_{0}^{\prime}(0)=0$ making equation (17) meaningless. However in this situations, finite size corrections are smaller, and so it should be possible to envisage a method to turn this point into advantage. This is easily achieved by applying the HCM equation (6) to the perturbation $b_{L}$ rather than to the energy density. In this case the solution of $(d+\zeta+1) \partial_{L} b_{L}(g)+L \partial_{L}^{2} b_{L}(g)=0$ gives

$$
g_{L}^{*, H C M}-g_{c}=\frac{(1-\nu \omega)(1-\nu(d+\zeta+\omega)) h(0) \Phi_{1}^{\prime}(0)}{[2(d+\zeta) \nu-4] \Phi_{0}^{\prime \prime}(0)} L^{-1 / \nu-\omega},
$$




\begin{tabular}{|c|c|c|c|}
\hline Method & $\Phi_{0}$ linear & $\Phi_{0}^{\prime}(0)=0$ & $\Phi_{0}^{\prime}(0)=0, \Phi_{1}^{\prime}(0)=0$ \\
\hline $\operatorname{FSCM}\left(e_{L}\right)$ & $1 / \nu$ & $1 / \nu-\omega$ or not applicable & not applicable \\
\hline $\operatorname{FSCM}\left(b_{L}\right)$ & $1 / \nu$ & $\min (2 / \nu, 1 / \nu+\omega)$ & $2 / \nu$ \\
\hline $\operatorname{HCM}\left(e_{L}\right)$ & $1 / \nu+\omega$ & $1 / \nu$ & not applicable \\
\hline $\operatorname{HCM}\left(b_{L}\right)$ & $1 / \nu$ & $1 / \nu+\omega$ & $2 / \nu+\omega$ \\
\hline $\operatorname{MHC}$ & $1 / \nu$ & $1 / \nu+\omega$ & $2 / \nu+\omega$ \\
\hline
\end{tabular}

Table 1. Summary of values of shift exponents in different cases. Only the minimum possible shift exponent is given. For instance, when also $\Phi_{1}^{\prime}(0)=0$, the shift exponent for $\operatorname{HCM}\left(b_{L}\right)$ (which means HCM applied to $b_{L}$ ) and MHC becomes at least $2 / \nu+\omega$. When the method formally returns a sequence that does not converge to the critical point in the limit $L \rightarrow \infty$ we have reported "not applicable".

and the shift exponent is $1 / \nu+\omega$. If also $\Phi_{1}$ does not contain a linear term the shift exponents becomes $2 / \nu+\omega$ when no other cancellations are present. The numerical test we have performed (see section 3.2 below) fall in this case (see also table 1). For completeness we note that, when $\Phi_{0}^{\prime}(0)=0$, the FSCM applied to the energy gives formally a shift exponent $1 / \nu-\omega$. This is clearly a poor convergence and the method can be successfully applied only when $1 / \nu>\omega$.

\subsection{The Berezinskii-Kosterlitz-Thouless transition}

The presence of the additional $\omega$ in $\theta_{H C M}$ suggests that the method could work even in the situation where formally $\nu=\infty$ like in the Berezinskii-Kosterlitz-Thouless (BKT) transition. In the BKT case the correlation length diverges with an essential singularity of the form $\xi \sim \exp \left(a t^{-\sigma}\right)$, where $a$ and $\sigma$ are constants. For instance in the classical two dimensional XY model $\sigma=1 / 2$. Using a modified Ansatz adapted to the BKT, one can show [19] that the HCM Eq. (6) still defines a sequence of pseudo-critical points converging to the true critical point with shift exponent $\theta_{H C M}^{B K T}=\epsilon /(n-1+\sigma)$ with $\epsilon>0$ and $n$ is an integer greater than one.

For the application of the HCM equation one simply requires the knowledge of $b_{L}$ which is the average of a (typically local) quantity over the ground state, and the dynamic exponent $\zeta$. We point out here that, contrary to PRG method (the current method of choice for estimating quantum critical points) no need of excited states is required. This is a big advantage since, in every numerical method excited states (if available) are typically obtained with smaller accuracy than the ground state. Instead the local average $b_{L}$ can be obtained with very high precision. In addition, the HCM is not restricted to quantum mechanical systems and is superior to the PRG in that it produces a faster converging sequence.

\subsection{The modified homogeneity conditions}

The dynamic exponent $\zeta$ is one in most known cases, however when not known a priori it can be easily found by looking at the gap behavior $\Delta=$ const. $\times L^{-\zeta}$ via exact diagonalization on small lattices. However in some cases (like two-dimensional quantum mechanics) assessing $\zeta$ can be a problem, but this requirement can in fact be lifted. Considering also the finite size sequence of the 
ground state energy $e_{L}$, it is possible to find a modified homogeneity condition (MHC) given by

$$
\frac{b_{L}\left(g_{L}^{*}\right)-b_{L-\delta L}\left(g_{L}^{*}\right)}{e_{L}\left(g_{L}^{*}\right)-e_{L-\delta L}\left(g_{L}^{*}\right)}=\frac{b_{L+\delta L}\left(g_{L}^{*}\right)-b_{L}\left(g_{L}^{*}\right)}{e_{L+\delta L}\left(g_{L}^{*}\right)-e_{L}\left(g_{L}^{*}\right)},
$$

or in the continuum version

$$
\frac{\partial_{L} b_{L}(g)}{\partial_{L} e_{L}(g)}=\frac{\partial_{L}^{2} b_{L}(g)}{\partial_{L}^{2} e_{L}(g)}
$$

Using the appropriate scaling Ansatz for the energy $e_{L}$ and the perturbation $b_{L}$ one can show, that the MCH Eq. (9) produces a pseudo-critical sequence $g_{L}^{*, M H C}=g_{c}+$ const. $\times L^{-\theta_{M H C}}$. If $\Phi_{0}$ has a linear term, the shift exponent is only $1 / \nu$, growing to $1 / \nu+\omega$ when $\Phi_{0}^{\prime}(0)=0$, which becomes $2 / \nu+\omega$ if also $\Phi_{1}^{\prime}(0)=0$.

\subsection{General prescription}

In table 1 we summarize the predicted shift exponents associated to different methods. Notice that only the minimal shift exponents are computed. When one has additional cancellations in the scaling functions, shift exponents tend to be higher. For example, when neither $\Phi_{0}$ or $\Phi_{1}$ contain a linear term, the shift exponent computed using the HCM and the MHC is $2 / \nu+\omega$. From the available information about the coefficients $\Phi_{0}^{\prime}(0)$ and $\Phi_{1}^{\prime}(0)$, possibly gathered by means of analytical arguments (symmetries, dualities, etc.) and/or graphical inspection of the numerical data, one should be able to use the best possible "recipe" to locate the critical point with the largest shift exponent. For instance, if $\Phi_{0}$ carries a linear term in the scaling variable, then the best strategy turns out to be the HCM applied to the energy density.

However, sticking to the HCM, one could also adopt a more "automatic" procedure, in the sense that both $e_{L}$ and $b_{L}$ are computed at once without any previous knowledge about the linear terms in the scaling functions. Then, one of the two following situations will occur: (i) $\operatorname{Both} \operatorname{HCM}\left(e_{L}\right)$ and $\operatorname{HCM}\left(b_{L}\right)$ converge to the same critical point (within numerical uncertainties), meaning that $\Phi_{1}^{\prime}(0) \neq 0$ and one has to refer to the second or third column of table 1 A best fit on the sequence with faster convergence then yields the exponent $1 / \nu+\omega$, while the other sequence gives $1 / \nu$ and so the two exponents $\nu$ and $\omega$ can be estimated independently. (ii) If instead $\operatorname{HCM}\left(e_{L}\right)$ and $\operatorname{HCM}\left(b_{L}\right)$ converge to different points (or the energy one does not converge at all), then one has to rely on the latter and the best fit returns $2 / \nu+\omega$ (fifth column of table 1). Even if now $\nu$ and $\omega$ are no longer separated, the convergence towards the critical point is enhanced by a larger exponent.

This summary concludes our theoretical analysis of the improved shift exponents based on the contributions beyond the scaling hypothesis. In the following we will consider practical implementations of the accelerated methods HCM and MHC and compare them against previous methods namely the PRG and the FSCM. We will consider an exactly solvable case where all the quantities can be calculated analytically, a non-integrable example and finally one instance of the extremely severe BKT case. In all cases the HCM and MHC represent an improvement with respect to previous methods and allow one to reduce to shorter sizes.

Our general strategy is that of obtaining accurate numerical values possibly free from uncontrollable errors, for short sizes. This can be achieved with Lanczos diagonalization on short sizes or DMRG 
keeping a large number of states and moderate sizes. In this way we avoid the pitfall of relying the extrapolated critical points on data which have potentially unknown errors as it may happen in quantum Monte Carlo or the so-called "infinite size" DMRG.

\section{Examples}

\subsection{An exactly solvable case: the $X Y$ model in transverse field}

To check our methods analytically we consider the one-dimensional $X Y$ model in transverse field [12]. It is given by the following Hamiltonian

$$
H=-\sum_{j=1}^{L}\left[\frac{(1+\eta)}{2} \sigma_{j}^{x} \sigma_{j+1}^{x}+\frac{(1-\eta)}{2} \sigma_{j}^{y} \sigma_{j+1}^{y}+h \sigma_{j}^{z}\right] .
$$

As usual we will employ PBC to avoid the appearance of surface corrections, and we will use $L$ even. The model Eq. (11) can be exactly solved by first passing to spinless fermion via Jordan-Wigner transformation and then diagonalizing the quadratic Hamiltonian with a suitable Bogoliubov transformation. It turns out that, for all $\eta \neq 0$, there is a critical point at $h=h_{c}=1$ which belongs to the same universality class as the two-dimensional classical Ising model. All quantities of interest like finite size energy density $e_{L}$ or perturbation $b_{L}$, and even lowest gap $\Delta_{L}$ can be computed exactly. In the quasi-critical regime $\xi \gg L$ one is able to find asymptotic expressions which are Laurent expansion in $L$. More details about the calculations can be found in [19], here we only quote the results. The energy density at finite size in the quasi-critical region has the following form

$$
\begin{aligned}
e_{L}(h, \eta) & =e_{\infty}(1, \eta)-\frac{\pi|\eta|}{6} L^{-2}-\frac{7 \pi^{3}}{360} \frac{\left(4 \eta^{2}-3\right)}{4|\eta|} L^{-4} \\
& +(h-1)\left[b_{\infty}(1, \eta)-\frac{\pi}{12|\eta|} L^{-2}-\frac{7 \pi^{3}\left(3-2 \gamma^{2}\right)}{2880|\eta|^{3}} L^{-4}\right] \\
& -\frac{(h-1)^{2}}{2} \frac{\ln (L)+\gamma_{C}+\ln (8|\eta| / \pi)-1}{\pi|\eta|}+O\left((h-1)^{3}\right) .
\end{aligned}
$$

The Ising model has $\alpha=0$ and a logarithmic singularity which at this level shows up in the $\ln L$ term at second order. The perturbation $b_{L}$ has a similar expansion in the quasi-critical region:

$$
\begin{aligned}
b_{L}(h, \eta) & =b_{\infty}(1, \eta)-\frac{\pi}{12|\eta|} L^{-2}-\frac{7 \pi^{3}\left(3-2 \gamma^{2}\right)}{2880|\eta|^{3}} L^{-4} \\
& -(h-1) \frac{\ln (L)+\gamma_{C}+\ln (8|\eta| / \pi)-1}{\pi|\eta|}+O\left(z^{2}\right) .
\end{aligned}
$$

The logarithmic singularity in principle requires a modified scaling Ansatz than that outlined in section 2 (see e.g. [4, 19]). However, stopping at first order, we can still compare this expression with our scaling Ansätze Eqns. (4) and (5) expanded in the quasi-critical region. We observe that, in this case $\Phi_{0}^{\prime}(0)=\Phi_{1}^{\prime}(0)=0$. We can also read off the function $F(h)$ (here $h$ plays the role of $g)$. Clearly $F(h)=-\pi|\eta| / 6-\pi(h-1) /(12|\eta|)+O(h-1)^{2}$. This is in perfect agreement with 
the conformal field theory (CFT) formula $F(h)=-\pi c v(h) / 6$ taking into account that the central charge is $c=1 / 2$ and the one particle dispersion is $\Lambda_{k}=2 \sqrt{\eta^{2}+h^{2}+\left(1-\eta^{2}\right) \cos (k)^{2}+2 h \cos (k)}$ giving rise to an $h$-dependent velocity $v(h)=2|\eta|+(h-1) /|\eta|+O(h-1)^{2}$ close to the critical point.

For what regards the terms of order $L^{-4}$, since $d+\zeta=2$ one deduces that $\omega=2$ (this exponent was also noted in 20]). Since $\nu=1$ one would conclude that this term arises from an irrelevant operator of dimension $(2+2)=4$. A deeper understanding of the finite size corrections of the $X Y$ model at the critical point $(h=1)$ can be obtained from the point of view of perturbed CFT. It was shown in 18] that the leading finite-size corrections of the critical $X Y$ model with PBC have scaling dimension 3 (this is an operator belonging to the tower of the energy density in CFT language) and 4 (tower of the identity). The operator with scaling dimension 3 would contribute to the energy density at finite size with a term of order $L^{-3}$. However at first order in perturbation theory the relevant matrix element vanishes identically, the first non-zero contribution is at second order giving rise to a $L^{-4}$ correction [18]. Summarizing, the leading corrections arising from irrelevant operators with scaling dimension 3 and 4 are both of order $L^{-4}$ in accordance with Eq. (12) for $h=1$.

After this short digression we can use Eqns. (12) and (13) to calculate shift exponents associated to different techniques. Let us consider the HCM first. By direct inspection one sees that in this case $\Phi_{0}^{\prime}(0)=\Phi_{1}^{\prime}(0)=0$. According to table 1 the HCM conditions are applicable with the perturbation $b_{L}$. Applying the HCM equations to Eq. (13) one obtains

$$
h_{L}^{*, H C M}=1+\frac{7 \pi^{4}\left(2 \eta^{2}-3\right)}{720 \eta^{2}} L^{-4}+\ldots
$$

telling us that $\theta_{H C M}=4$. Since, from the exact solution one has $\nu=1$ and we just recovered $\omega=2$, this result is consistent with $\theta_{H C M}=\omega+2 / \nu$ valid when $\Phi_{0}^{\prime}(0)=\Phi_{1}^{\prime}(0)=0$. The modified method MHC gives instead

$$
h_{L}^{*, M H C}=1-\frac{7 \pi^{4}\left(2 \eta^{4}+\eta^{2}-3\right)}{720 \eta^{2}} L^{-4}+\ldots
$$

which again confirms $\theta_{M H C}=\theta_{H C M}=4$ for this case. In this model we can also find exactly the shift exponent related to the PRG method. All we need is the expression for the lowest gap at finite size, close to the critical point which we recall here from [19]

$$
\begin{aligned}
\Delta_{L} & =(h-1)+\frac{\pi\left(2 \eta^{2}+h-1\right)}{4|\eta|} L^{-1} \\
& +\frac{\pi^{3}\left[3(h-1)-2(2+h) \eta^{2}+8 \eta^{4}\right]}{192|\eta|^{3}} L^{-3}+O\left(L^{-5}\right) .
\end{aligned}
$$

Since $d=\zeta=1$ the PRG method consists of imposing $\partial_{L}\left[L \Delta_{L}\left(h_{L}^{*}\right)\right]=0$, which yields [19]

$$
h_{L}^{*, P R G}=1+\frac{\pi^{3}\left(4 \eta^{2}-3\right)}{48|\eta|} L^{-3}+\ldots
$$

Finally the FSCM gives $h_{L}^{*}=1+L^{-2} \pi^{2} / 6$, in accordance with $\theta_{F S C M}=2 / \nu$. 


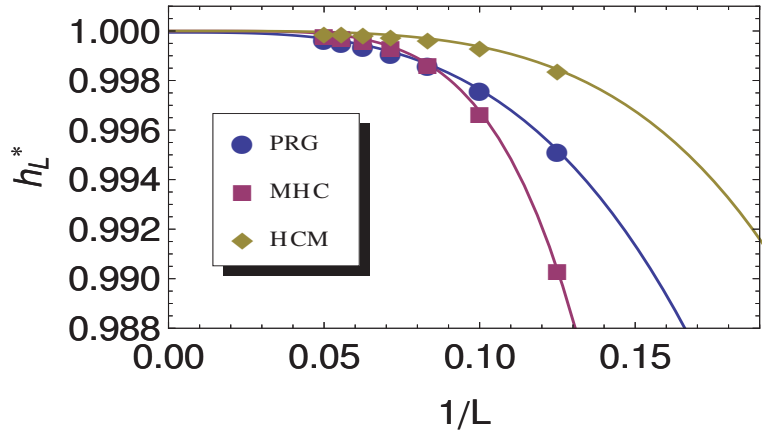

Figure 1. (color on-line) Sequences of pseudo-critical points obtained with the PRG, the MHC [given by Eq. [9] ] and HCM [Eq. [8] ]. Continuous lines are algebraic best-fits to the data of the form $h_{L}^{*}=h_{c}+A L^{-\phi}$.

\begin{tabular}{|c|c|c|c|}
\hline Method & Estimated $h_{c}$ & Error & $\phi$ \\
\hline PRG & $0.99994(2)$ & $9 \times 10^{-6}$ & 3.24 \\
\hline $\operatorname{HCM}\left(b_{L}\right)$ & 1 & $7 \times 10^{-8}$ & 4.03 \\
\hline MHC & 1 & $2 \times 10^{-5}$ & 4.88 \\
\hline
\end{tabular}

Table 2. Summary of estimated critical points and error for the $X Y$ model in transverse field. The anisotropy has been fixed to $\eta=0.5$. Pseudo-critical sequences are obtained using finite size data from $L=8,10,12, \ldots, 20$. The critical point is obtained using a non-linear fit of the form $h_{L}^{*}=h_{c}+A L^{-\phi}$. The standard error of the regression is defined according to $\left((N-p)^{-1} \sum_{j=1}^{N}\left(y_{j}-f\left(x_{j}\right)\right)^{2}\right)^{1 / 2}$ where $\left(x_{j}, y_{j}\right)$ are the data points, the fitted function is $f(x)=h_{c}+A x^{\phi}$ and the number of parameters of the fit $p$ is 3 in our case.

All in all these explicit calculations confirm that the accelerated HCM and MHC methods are superior to the PRG or the FSCM methods.

In this exactly solvable case we can also see test the efficiency of the various methods using finite size data on small sizes. Using finite size data for $L=8,10,12, \ldots, 20$ we compute sequences of pseudo-critical points for the PRG, MHC and HCM methods. We then estimate the critical point using a non-linear fit of the form $h_{L}^{*}=h_{c}+A L^{-\phi}$, with $h_{c}, A, \phi$ unknown. The results of the fit are plotted in Fig. 1 while the estimated critical point and errors are summarized in Table 2.

\subsection{Numerical example: $c=1$ transition}

We consider here a spin-1 model with anisotropies given by the following Hamiltonian

$$
H=\sum_{j=1}^{L}\left[S_{j}^{x} S_{j+1}^{x}+S_{j}^{y} S_{j+1}^{y}+J_{z} S_{j}^{z} S_{j+1}^{z}+D\left(S_{j}^{z}\right)^{2}\right],
$$




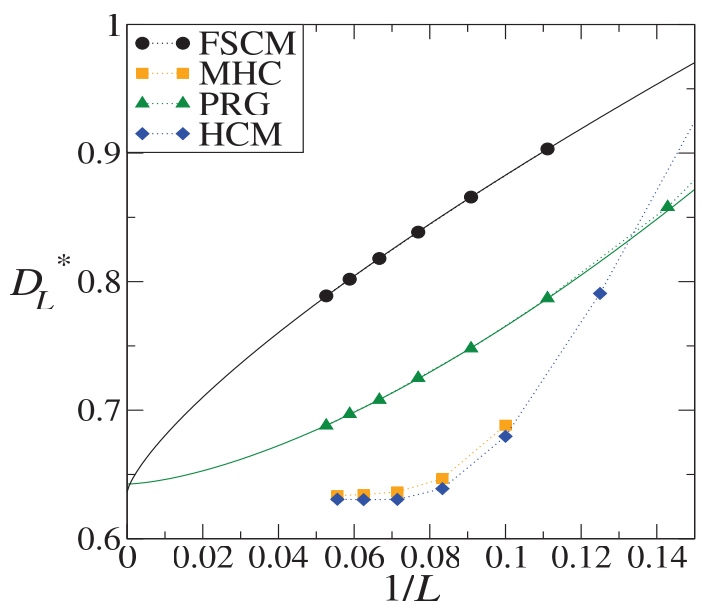

Figure 2. (color on-line) The sequences of pseudo-critical points obtained with the FSCM, the PRG and new methods HCM (given by Eq. (8)) and the MHC Eq. (9). Continuous lines are algebraic best-fits to the data.

where as usual, PBC are used. The Hamiltonian Eq. (15) has been used to describe magnetic properties of different quasi 1D compounds and an extensive literature is available (see e.g. Refs. [10, 13, 14] and reference therein). We fix here to the value $J_{z}=0.5$ where a critical point of order $D_{c}=0.6 \div 0.7$ has been estimated previously. At this particular transition point it has been previously found [13] a pretty large value of the correlation length exponent $\nu=2.38$, which implies a slow scaling in $1 / \nu$.

The advantage of having fast converging sequences is that one can limit the numerical calculations to short lattice sizes that can be reached by exact diagonalization. To illustrate our procedures we used exact Lanczos diagonalization for chains of length $L=8,10,12,14,16$ and used high precision DMRG simulations (with as many as $3^{7}$ optimized states and many zips) only for chain length $L=18,20$. The role of $b_{L}$ is given here by $b_{L}=\left\langle\left(S_{i}^{z}\right)^{2}\right\rangle$. We tested different methods: the FSCM, PRG, HCM, and MHC. We first observed that the energy curves at different size do not cross. This means that there are no linear terms in the scaling functions and consequently we applied the FSCM and the HCM to the perturbation operator $b_{L}$. The results are plotted in Figure 2, It is apparent that the HCM and MHC result in much more rapidly converging sequences respect to other methods, including the PRG.

The extrapolated critical points together with the relative shift exponents for different methods, are summarized in Table 3. In fact by inspecting the finite-size critical points we see that for HCM and MHC the values of $D_{c}(L)$ at the largest plotted sizes already are saturated within the error bar induced by the sampling, $\delta D=0.1$, that propagates to $O\left(\delta D^{3}\right)$ when the zeroes/crossings are located using cubic splines (an error-free localization of zeroes would require adaptive sampling with increasing computational load). At this stage, if we insist on computing a shift exponent, we can use a fit in which the infinite-size critical point is kept fixed as in Table 3 and in this way we estimate $\theta_{H C M}, \theta_{M H C} \geq 8$. As far as FSCM and PRG are concerned, the nonlinear best-fit curves underlying the data in Table 3 are also displayed in Figure 2 . 


\begin{tabular}{|c|c|c|c|c|}
\hline & FSCM & PRG & HCM & MHC \\
\hline$D_{c}$ & $0.638 \pm 0.006$ & $0.643 \pm 0.003$ & $0.631 \pm 0.001$ & $0.634 \pm 0.001$ \\
\hline fitted $\theta$ & 0.75 & 1.54 & $\geq 8$ & $\geq 8$ \\
\hline
\end{tabular}

Table 3. Extrapolated critical points together with shift exponents for various methods.

\subsection{BKT transition}

To finish our series of numerical test we consider the example of a BKT transition. In such a transition the gap opens up so slowly (remind $\Delta \sim \xi^{-1} \sim \exp \left(-a t^{-\sigma}\right)$ ) that the PRG method is practically not applicable. As an example of BKT transition we consider the spin- $1 / 2$ Heisenberg model with frustration due to next-to-nearest neighbors interaction:

$$
H=\sum_{j=1}^{L} J_{1} \vec{S}_{j} \cdot \vec{S}_{j+1}+J_{2} \vec{S}_{j} \cdot \vec{S}_{j+2} .
$$

The model is equivalent to a 2 -legs zigzag ladder with $L / 2$ rungs. We will also fix $J_{1}=1$ for clarity. The model is gapless for $J_{2}<J_{2 c}$ while it has a doubly degenerate ground state for $J_{2}>J_{2 c}$. The most precise estimate for the critical point was done by Okamoto and Nomura in [16] using a model-specific method where the crossing points are determined by the coincidence of the gaps in the singlet and the triplet sectors. They found $J_{2 c}=0.2411 \pm 0.0001$, which sets the "accepted value" to date. The role of the parameter $g$ is played here by $J_{2}$ while the perturbation operator is given by $b_{L}=\left\langle\vec{S}_{j} \cdot \vec{S}_{j+2}\right\rangle$. The numerical calculations were done using ladders with PBC and up to $L / 2=13$ rungs, an effective $\delta L=2$, and a number of DMRG states large enough to guarantee an exact value of the ground state energy up machine precision. Here we have chosen to determine adaptively the mesh of values for $J_{2}$ in order to detect the zero-crossing points with the best precision.

We now pass to examine the different methods. As already mentioned the PRG is not feasible because of the extremely slow opening of the gap. The FSCM also fails as it turns out to be impossible to find a real solution to the FSCM equation. Instead the HCM shows a sequence of zero-crossing points, as displayed in the upper panel of Figure 3 This sequence, plotted in the lower panel of Figure 3 as a function of $1 / L$, converges to a finite $J_{2}$ with a shift exponent close to one. A linear fit gives $J_{2 c}=0.2610 \pm 0.0004$. Such a critical value is slightly larger than the one reported in [16], but it is definitely different to it as the small error bars exclude compatibility between the two. According to the present numerical analysis, our method predicts a larger critical phase $J<J_{2}$, where the quantity $b(L)$ is found to be homogeneous of degree 2 , a feature that is associated with a critical phase.

In principle, both the HCM method and the one proposed by Okamoto and Nomura should converge to the same critical point, no matter how fast they attain the asymptotic value for $L \rightarrow \infty$. This discrepancy raises an interesting puzzling question on how to establish the right methods to determine the correct location of the BKT transition. Let us mention that the crossing method in [16] is ruled by the additional assumption that in the RG scheme the bare coefficient of the marginal term vanishes at the BKT point. However, irrelevant terms at criticality may introduce a finite contribution to this coefficient, introducing a possible source of systematic deviation from the exact critical value. In any case, the solution of this challenging problem seems to need a thorough analysis close to a criticality, with a careful treatment of any possible logarithmic correction that 

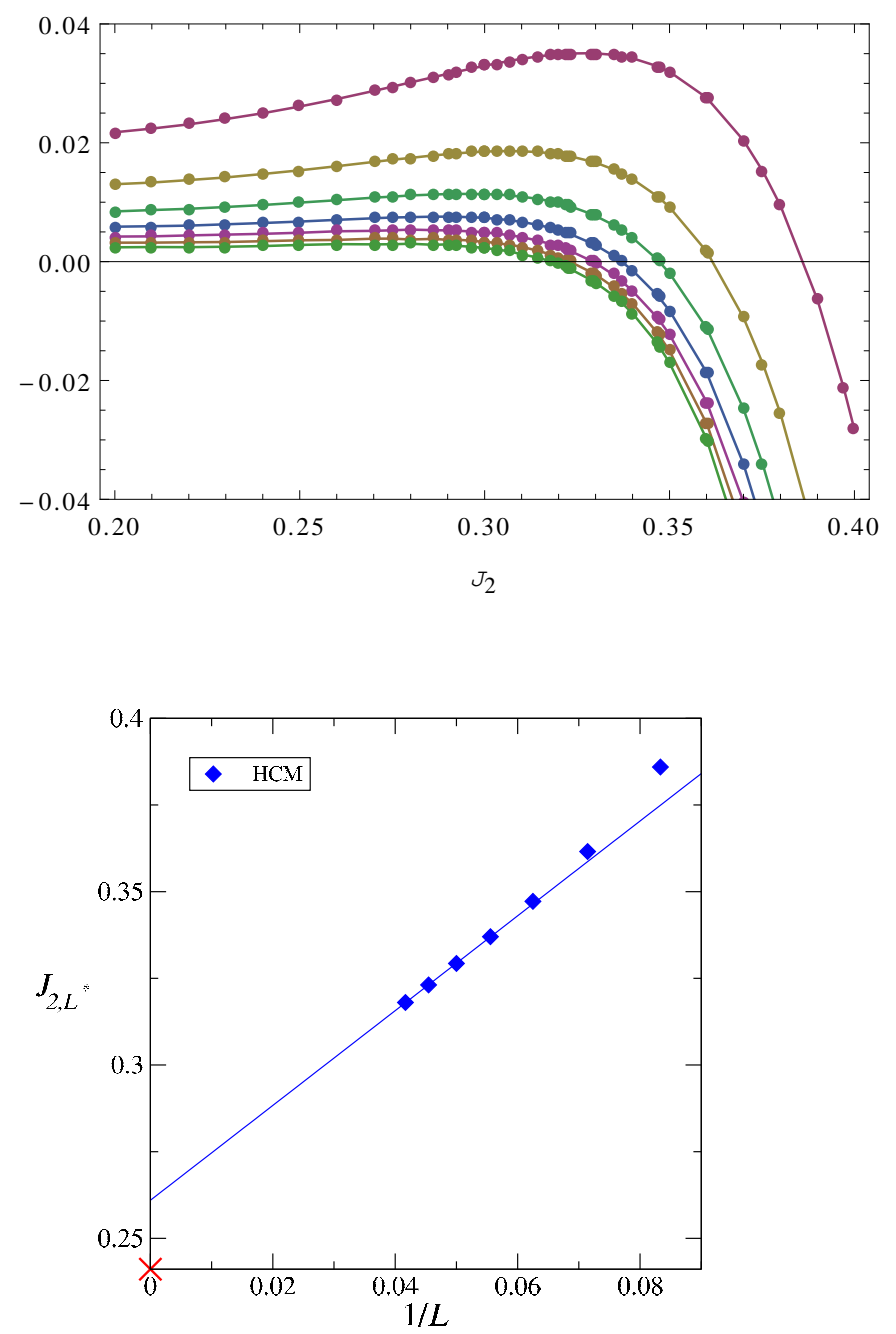

Figure 3. (color on-line) Upper panel: Search for the zeros of Eq. (8) with $\delta L=2$. Lower Panel: Extrapolations of the pseudo-critical points $J_{2, L}^{*}$. For reference, we have marked with a red cross the value 0.2411 calculated in Ref. [16] (see text for details).

is typically associated to transitions of BKT type. We think that this task deserves a dedicated study and it is clearly beyond the scopes of the present article. Here, we limit ourselves to report on the success of the HCM method to converge remarkably fast to a finite critical value for the BKT transition, a problem known to be extremely severe to treat. 


\section{Conclusions}

Locating the critical point is one of the first issues which arise when considering a statistical mechanical problem. Available methods to extract critical points from numerical simulations are, among others: i) looking for the maxima of a susceptibility ii) intersection points of reduced (Binder's) cumulants at different system sizes, iii) look for closing of gaps in quantum systems (PRG). These methods produce sequences of pseudo-critical points $g_{L}^{*}$ which converge to the true critical point as $g_{L}^{*}=g_{c}+O\left(L^{-\theta}\right)$ with a leading algebraic term controlled by the shift exponent $\theta$. The general belief is that the shift exponent is given by the inverse of the correlation length exponent: $\theta=1 / \nu$. In this article we have developed accelerated methods for the location of critical

points. According to the present scaling analysis, our methods can in principle be applied at any second order transition in any dimension quantum or classical, temperature or parameter driven. The resulting pseudo-critical sequences are characterized by a large value of the shift exponent which enables these methods to be efficient even in the extremely hard case of BKT transition where formally " $\nu=\infty "$.

The advantage of having such methods at disposal, is that one can limit the analysis to much shorter system sizes. In this paper, we have shown how the proposed methods are able to produce fast converging sequences in $1 \mathrm{D}$, focussing on three problems of different nature: the exactly solvable XY model, a non integrable spin- 1 model and the frustrated spin- $1 / 2$ Heisenberg model. We have shown that the value of the critical point is correctly determined in the first case, and in the second it is estimated with high accuracy. Interestingly, the Homogeneity Condition Method is able to produce fast converging sequences in correspondence of the Berezinskii-Kosterlitz-Thouless transition for the third test model, but the critical point value differs from the previous estimation given in Ref. [16]. As both values are coming from scaling assumptions predicted by a renormalization group analysis, we take this discrepancy as a challenging problem to address in future studies. Finally, we think that our methods can find important applications in two dimensional quantum mechanical problems with frustration, for which quantum Monte Carlo methods are plagued with the sign problem and the reliable numerical data are therefore restricted to very short sizes.

\section{Acknowledgments}

MR acknowledges support from Compagnia di san Paolo.

\section{References}

[1] Affleck I 1986 Phys. Rev. Lett. 56, 746.

[2] Aharony A and Fisher M E 1983 Phys. Rev. B 27, 4394.

[3] Amico L, Fazio R, Osterloh A and Vedral V 2008 Rev. Mod. Phys. 80, 517.

[4] Barber M N 1983 in C Domb and J. L Lebowitz, eds, 'Phase transitions and critical phenomena' Vol. 8 Academic press.

[5] Barton G and Dombey N 1984 Nature 331, 336.

[6] Baxter R J 1982 Exactly Solved Models in Statistical Mechanics Pergamon Oxford.

[7] Binder K 1981 Z. Phys. B 43, 119. 
[8] Campos Venuti L, Degli Esposti Boschi C, Roncaglia M and Scaramucci A 2006 Phys. Rev. A 73, 010303(R).

[9] Campos Venuti L and Zanardi P 2007 Phys. Rev. Lett. 99, 095701.

[10] Chen W, Hida K and Sanctuary B C 2003 Phys. Rev. B 67, 104401.

[11] Fisher M E and Barber M N 1972 Phys. Rev. Lett. 28, 1516.

[12] Lieb E, Schultz T and Mattis D 1961 Ann. Phys. 16, 407.

[13] Degli Esposti Boschi C, Ercolessi E, Ortolani F and Roncaglia M 2003 Eur. Phys. J. B 35, 465.

[14] Degli Esposti Boschi C and Ortolani F 2004 Eur. Phys. J. B 41, 503.

[15] Nightingale M P 1975 Physica A 83, 561.

[16] Okamoto K and Nomura K 1992 Phys. Lett. A 169, 433.

[17] Patasinskij A Z and Pokrovskij V L 1979 Fluctuation theory of phase transitions Pergamon Press Oxford.

[18] Reinicke P 1987 J. Phys. A: Math. Gen. 20, 5325.

[19] Roncaglia M, Campos Venuti L and Degli Esposti Boschi C 2008 Phys. Rev. B 77, 155413.

[20] Salas J and Sokal A D 2000 J. Stat. Phys. 98, 551. arXiv:cond-mat/9904038v1.

[21] Wegner F J 1972 Phys. Rev. B 5, 4529.

[22] Zanardi P, Giorda P and Cozzini M 2007 Phys. Rev. Lett. 99, 100603.

[23] Zanardi P, Paris M G A and Campos Venuti L 2008 Phys. Rev. A 78, 042105. 